\title{
Prospective of Fifth Generation Mobile Communications
}

\author{
Dr. Anwar M. Mousa \\ University of Palestine,Gaza- Palestine \\ a.mousa@up.edu.ps
}

\begin{abstract}
This paper explores future mobile systems with emphasis on re-configurability based on cognitive and software defined radios. 5G (Fifth Generation) network architecture consisting of reconfigurable multitechnology core and a single fully reconfigurable terminal able to autonomously operate in different heterogeneous access networks is proposed. The proposed network is enforced by nanotechnology, cloud computing and based on All IP Platform. The paper highlights $5 G$ main development challenges and illustrates why there is a need for 5G. It also reviews in brief the evolution of wireless and cellular systems focusing on four main key factors: radio access, data rates, bandwidth and switching schemes in addition to change in network architecture. The $3 G$ transitional cellular and wireless systems toward $4 G$ and the true $4 G$ IMT-advanced systems are thoroughly presented.
\end{abstract}

\section{Keywords:}

Re-configurability; cognitive radio; software defined radio; $5 G$ mobile communications; nanotechnology; cloud computing; network architecture.

\section{Introduction}

Cellular generations differ, in general, in four main aspects: radio access, data rates, bandwidth and switching schemes. The $1 \mathrm{G}$ (First Generation) cellular systems, mainly analog system, had a bandwidth ranging from 10 to $30 \mathrm{KHz}$ depending on system type and service. Offered data rates were around $10 \mathrm{Kbps}$ after analog to digital conversion. Radio access scheme was FDMA and switching was all circuit, suitable for voice services. The first phase of the $2 \mathrm{G}$ (Second Generation) GSM systems offered a data rate up to $9.6 \mathrm{Kbps}$ and increased in the second phase and phase+ to reach a peak rate of more than $300 \mathrm{Kbps}$ with bandwidth of $200 \mathrm{KHz}$ [1], [2]. Switching started to be packet in addition to circuit beginning from the second phase and radio access was TDMA/FDMA. For the 3G (Third Generation) systems, the peak data rate began of 2 Mbps in the first phase and approached 50Mbps in consecutive phases at constant wide bandwidth of $5 \mathrm{MHz}$ [3]. The approved access scheme for the 3G was CDMA and switching continued to be circuit in addition to packet. However, at the start of 3.5G, with HSDPA system [4], and thereafter it was focused on packet switching only. In 4G (Fourth Generation) cellular systems, peak data rates started at $100 \mathrm{Mbps}$ [5] and supposed to reach the order of more than 1 Gbps at the downlink benefiting from a variable bandwidth up to 20, 40 or even $70 \mathrm{MHz}$ [6]. Switching was approved to be packet only- all IP, and radio access changed from CDMA to OFDMA and SC-FDMA. In addition to the cellular systems, current wireless technologies include Wireless Local Area Networks (WLAN) 802.11 [7] and Wireless Metropolitan Area 
International Journal of Next-Generation Networks (IJNGN) Vol.4, No.3,September 2012

Networks (WMAN) 802.16 [8]. Moreover, ad-hoc Wireless Personal Area Network (WPAN) and wireless networks for digital TV are gaining more interest. Future generations will include new systems such as broadband wireless access systems, intelligent transport systems, high altitude platform station systems and millimeter-wave Local Area Networks. Key to the future generations of mobile communications are multimedia communications, wireless access to broadband fixed networks, and seamless roaming among different systems.

In 4G mobile systems different access technologies, such as WLAN, WMAN and cellular, are combined on a common platform and interoperate to offer different service in different radio environments [9]. On the other hand, the 5G (Fifth Generation mobile and wireless networks) can be a completed wireless communication without limitation, which bring us perfect real world wireless - World Wide Wireless Web (WWWW). 5G is a name used in some research papers and projects to denote the next major phase of mobile telecommunications standards beyond the 4G/IMT-Advanced standards. At present, $5 \mathrm{G}$ is not a term officially used for any particular specification or in any official document yet made public by telecommunication companies or standardization bodies such as 3GPP [10], WiMAX Forum [11], or ITU-R [12]. The rest of the paper is organized as follows: Section 2 reviews in brief the evolution of wireless and cellular systems focusing on the four main key factors: radio access, data rates, bandwidth and switching schemes in addition to change in network architecture. The 3G transitional cellular and wireless systems toward 4G and the true 4G IMT-advanced systems comprising mainly LTE advanced and Mobile WiMAX advanced are thoroughly presented. Section 3, constituting the main body of the research, explores the expected future mobile system with emphasis on why there is a need for 5G. It highlights $5 \mathrm{G}$ main development challenges and presents a proposed 5G network architecture. Section 4 concludes the paper.

\section{Evolution of Wireless and Cellular Systems}

This section reviews in brief the evolution of wireless and cellular systems focusing on the four main key factors: radio access, data rates, bandwidth and switching schemes in addition to change in network architecture.

\subsection{Review of Ex-Fourth Generations Systems}

\subsubsection{First-Generation Systems (1G)}

The first-generation cellular systems were introduced in the beginning of 1980's where almost all of them were analog systems using the frequency modulation technique for radio transmission. Traffic was multiplexed onto an FDMA system. The needs for improved transmission quality, higher system capacity, better system coverage, more services, security and better spectral efficiency paved the way toward second generation cellular systems.

\subsubsection{Second Generation Systems (2G)}

In Europe and most of the rest of the world, the Global System for Mobile communications (GSM) [13] gained the most popularity as $2 \mathrm{G}$ digital system with data transmission up to 9.6 kbps. GSM was introduced in 1990 and then evolved in 1995 with General packet radio service (GPRS) [1] where GPRS is a radio technology for GSM networks that adds packet-switching 
International Journal of Next-Generation Networks (IJNGN) Vol.4, No.3,September 2012 protocols. GPRS network is considered as $2.5 \mathrm{G}$ and differs from GSM architecture, Figure1, by the addition of two new network elements, see Figure 2:

- The Serving GPRS Support Node (SGSN): performs security functions, mobility management and access control. SGSN resides at the same hierarchical level as a visited MSC (VMSC)/VLR

- $\quad$ The Gateway GPRS Support Node (GGSN): used for inter-working with external packet-switched networks. GGSN has functions comparable to a gateway MSC (GMSC) and performs comparable functions such as routing and mobility management.

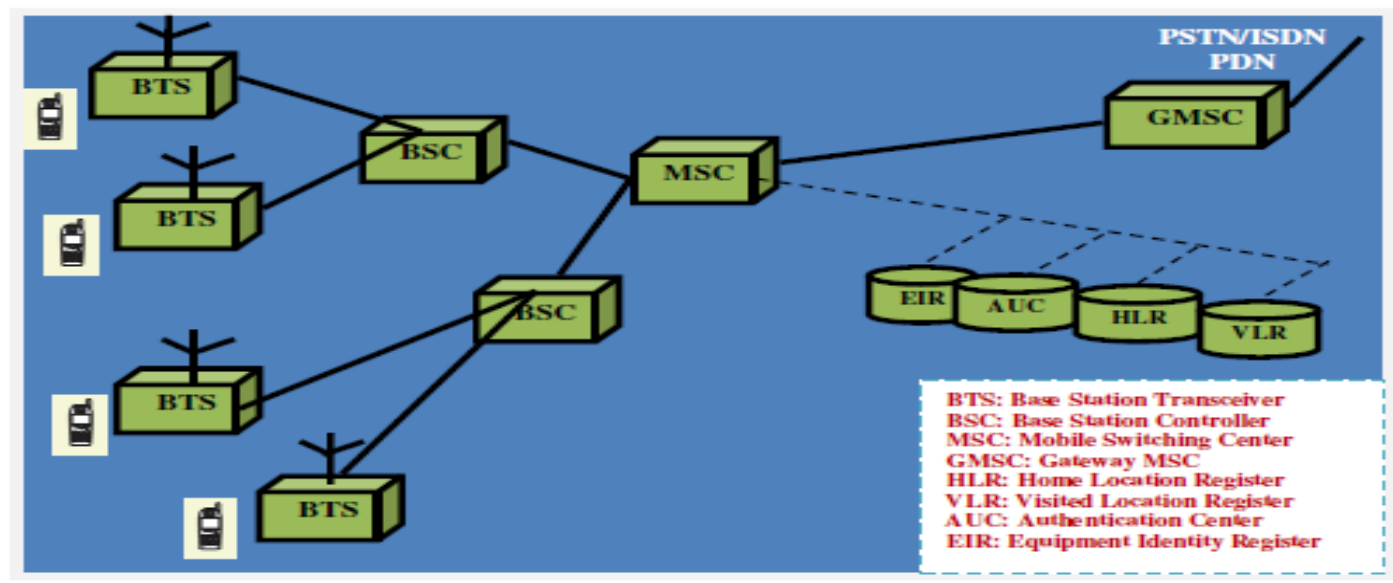

Figure 1: General GSM Network Architecture.

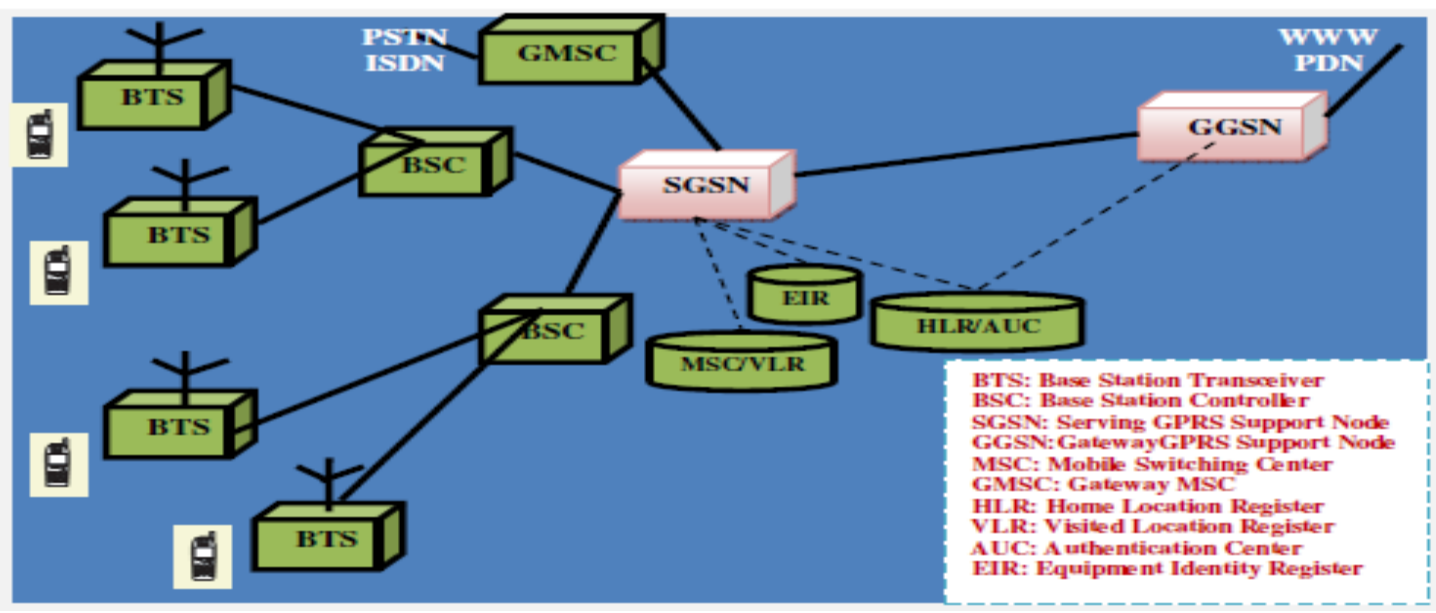

Figure 2: General GPRS Network Architecture.

Four Coding Schemes (CS1, CS2, CS3 and CS4) exist for GPRS with the highest (CS4) requires excellent radio signal (Carrier to Interference ration of $27 \mathrm{~dB}$ ) and delivers a user throughput of up to $21.4 \mathrm{kbits} / \mathrm{s}$ per time slot, allowing theoretical peak data rate of $171.2 \mathrm{kbits} / \mathrm{s} ; 8$ timeslots multiplied by 21.4. However, to reach the maximal transmission data rate a single user must utilize all eight slots. This is unrealistic in a practical GPRS installation; hence the actual data rate is much lower. GPRS allows the efficient use of scarce radio/time resources among a larger 
International Journal of Next-Generation Networks (IJNGN) Vol.4, No.3,September 2012

number of users but suffers from severe transit delays as packets are sent in different directions to reach the same destination causing packets loss or corruption.

The Enhanced data rates for GSM evolution (EDGE) [14] was developed to pave the way for Third-Generation Systems. The EDGE, considered as $2.75 \mathrm{G}$, allows $2 \mathrm{G}$ operators to use existing $2 \mathrm{G}$ radio bands to offer wireless multimedia IP-based services and applications and compete with $3 \mathrm{G}$ operators by offering similar data services. Theoretical speeds can reach more than $470 \mathrm{kbps}$ with a bit-rate of $59.2 \mathrm{kbps}$ per timeslot in good radio conditions. EDGE uses the same TDMA frame and channel bandwidth as GSM networks however, it uses (8-PSK), rather than normal GSM (GMSK) used by GSM or GPRS. To upgrade from GPRS, one EDGE transceiver unit is to be added to each cell and remote software updates is received by the base stations. This is the main difference in network architecture with that of GPRS. In USA, three standards for $2 \mathrm{G}$ systems, different than those in Europe, exist: (TDMA) based standard (IS-136), (CDMA) based standard (IS-95) and GSM derivative, Personal Communication Services (PCS) 1900.

\subsubsection{Third Generation (3G) Systems}

\subsubsection{UMTS}

The most important International Mobile Telecommunications-2000 (IMT-2000) [15] proposals are the UMTS (W-CDMA) [16] as the successor to GSM. UMTS licenses have been awarded across Europe and Asia through 3GPP group. For interoperability with North American networks, another group known as 3GPP2 was formed to develop global specifications for $3 \mathrm{G}$ networks. An assembly of international operators known as the Operator Harmonization Group (OHG) suggested the harmonization of the 3GPP and the 3GPP2 concepts, hence developing a Global Third Generation (G3G) to allow the interoperability of networks worldwide. UMTS [17] differs from GSM Phase $2+(2.5$ and $2.75 \mathrm{G})$ mostly in the new principles for air interface transmission, (W-CDMA instead of TDMA/ FDMA). The air interface access for User Equipment (UE) is provided by the UMTS Terrestrial Radio Access Network (UTRAN).

Two new network elements were introduced in UTRAN: RNC (Radio Network Controller) and Node B, Figure 3. The RNC is connected to a set of Node B elements, each of which can serve one or several cells. Channel access to UE is provided by UTRAN and the core network architecture of UMTS is based on GSM core with GPRS upgrades. However, all equipment has to be modified for UMTS operation and services. The core network is divided in circuit switched and packet switched domains. Some of the circuit switched elements are (MSC), (VLR) and Gateway MSC. Packet switched elements are (SGSN) and (GGSN). Other network nodes such as EIR, AUC and HLR, are shared by both domains. The core network performs switching and transmission functions and contains the databases and network management functions.

$3 \mathrm{G}$ systems offer high data rates up to $2 \mathrm{Mbps}$, over $5 \mathrm{MHz}$ channel carrier width, depending on mobility/velocity, and high spectrum efficiency. The data rate supported by $3 \mathrm{G}$ networks depends also on the environment the call is being made in; $144 \mathrm{kbits} / \mathrm{s}$ in satellite and rural outdoor, 384 $\mathrm{kbits} / \mathrm{s}$ in urban outdoor and $2048 \mathrm{kbits} / \mathrm{s}$ in indoor and low range outdoor. The two duplex modes; (TDD) and (FDD) [18] are supported by UMTS Terrestrial Radio Access implementing TDD for micro and pico cells and FDD for macro cells. 


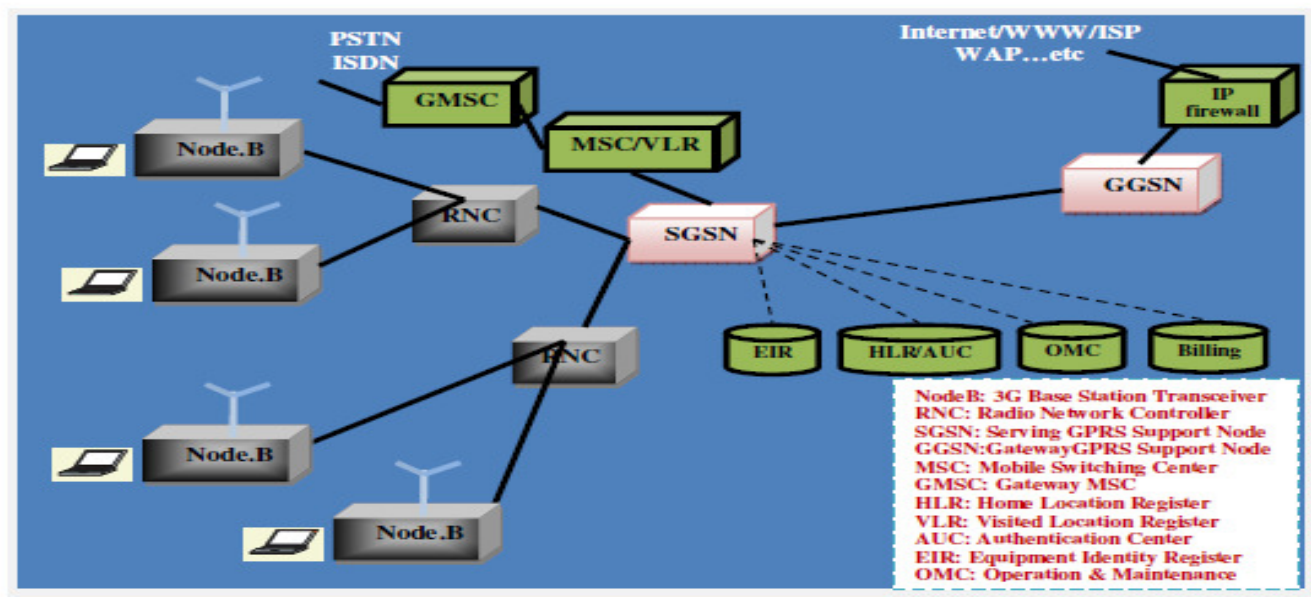

Figure 3: General UMTS Network Architecture.

\subsubsection{HSDPA- High Speed Downlink Packet Access}

HSDPA (Release 5 evolution of UMTS [4]) is considered as 3.5G with offered peak rate of 14.4 Mbps (with $2 \times 2$ antennas) in $5 \mathrm{MHz}$ channel at the downlink. Peak data rate could be more dependant on the number of antennas on transmit and receive antennas; in 3GPP release 7, named HSPA (High-Speed Packet Access) Evolved, antenna array technologies was introduced such as beam-forming and Multiple Input Multiple Output (MIMO), achieving data rates of up to 42 Mbps. The new technologies in HSDPA include:

- $\quad$ Short frame length; (Static TTI Length of 3 Time Slots = 2ms) to further accelerate packet scheduling for transmission

- New transport channel type; known as High Speed Downlink Shared Channel (HS$\mathrm{DSCH})$ to facilitate air interface channel sharing between several users

- $\quad$ Fast retransmissions; the request is processed in (Node-B) providing the fastest possible response. Incremental redundancy is also used for minimizing the air-interface load caused by retransmissions

- $\quad$ Fast scheduling; scheduling of the transmission of data packets over the air interface is performed in (Node-B) based on: channel quality, terminal capability, QoS class and power/code availability. Moreover, a short frame length is used, allowing scheduling to be as fast as possible.

- $\quad$ Adaptive Modulation and Coding-AMC; the modulation schemes and coding are udapted according to radio link quality. While the coding rate can vary between $1 / 4$ and $3 / 4$, the spreading factor remains fixed.

- $\quad$ Hybrid Automatic Repeat Request-H-ARQ; H-ARQ mechanism reduces the delay and increases retransmission efficiency.

For the uplink, a 3G mobile telephony protocol in the High-Speed Packet Access (HSPA) family was included in UMTS Release 6 standard published by 3GPP. It is referred as High-Speed Uplink Packet Access (HSUPA) [19] with Up-link speeds up to $5.76 \mathrm{Mbit} / \mathrm{s}$. After HSDPA, roadmap leads to HSOPA (High-Speed OFDMA Packet Access); the project, called the LTE 
International Journal of Next-Generation Networks (IJNGN) Vol.4, No.3,September 2012

(Long Term Evolution) initiative, achieved data rates of up $200 \mathrm{Mbps}$ for downlink and 100 Mpbs for uplink using OFDMA modulation.

\subsubsection{HSOPA- High Speed OFDM (Orthogonal Frequency Division Multiplexing) Packet Access [4][20].}

HSOPA is a proposed part of 3GPP's Long Term Evolution (LTE) [21], also often referred to as Super $3 \mathrm{G}$ or $3.75 \mathrm{G}$. HSOPA succeeds HSDPA and HSUPA technologies specified in 3GPP releases 5 and 6 . The main HSOPA Features are:

- Unlike HSDPA or HSUPA, HSOPA is an entirely a new air interface system (OFDMA), unrelated to and incompatible with W-CDMA.

- $\quad$ Flexible bandwidth usage with $1.25 \mathrm{MHz}$ to $20 \mathrm{MHz}$ bandwidths. By comparison, W-CDMA uses fixed size $5 \mathrm{MHz}$.

- Increased spectral efficiency at 2 to 4 times more than in 3GPP release 6

- Latency times of around $20 \mathrm{~ms}$ for round trip time from UE to RAN, much better than "classic" W-CDMA.

The reasons for upgrading UMTS to HSOPA are the emergence of competitive technologies, such as WiMAX [22] and the minimal cost to evolve a UMTS network to a next generation air interface, including HSOPA, compared to the cost of deploying a new network. Most of the existing infrastructure remains the same, requiring only major upgrades at a tower level and on handsets. HSOPA uses OFDM and MIMO antenna technology. It supports up to 10 times as many users as W-CDMA based systems and needs lower processing power required on each handset.

\subsubsection{G Transitional Systems}

The 3G transitional cellular and wireless systems toward 4G comprise mainly LTE (3GPP family) and Mobile WiMAX (IEEE family, 802.16e). The main features of both are analyzed as follows:

\subsubsection{Long-Term Evolution (LTE)}

The first release of LTE (release 8) was labeled as "3.9G". The goal was to provide a high-datarate, low-latency and packet-optimized radio access technology supporting flexible bandwidth deployments [23]. In order to allow seamless mobility with minimal latency, new network architecture was designed. The LTE system supports mobility for speed up to $350 \mathrm{~km} / \mathrm{h}$ with some performance degradation [24] but it is primarily optimized for much lower speeds (up to 15 $\mathrm{km} / \mathrm{h}$ ). LTE supports downlink peak data rates of $326 \mathrm{Mb} / \mathrm{s}$ with $4 \times 4 \mathrm{MIMO}$ (multiple input multiple output) within $20 \mathrm{MHz}$ bandwidth.

The LTE network architecture, Figure 4, results in a highly simplified flatter architecture with only two types of node - contrasting to many network nodes in the current network architecture of the $3 \mathrm{G}$ system- namely:

- evolved Node-B (eNB)

- Mobility Management Entity/Gateway (MME/GW) 
International Journal of Next-Generation Networks (IJNGN) Vol.4, No.3,September 2012

The radio network controller (RNC) is eliminated from the access network and its functions are implemented in multiple eNB yielding reduced latency.

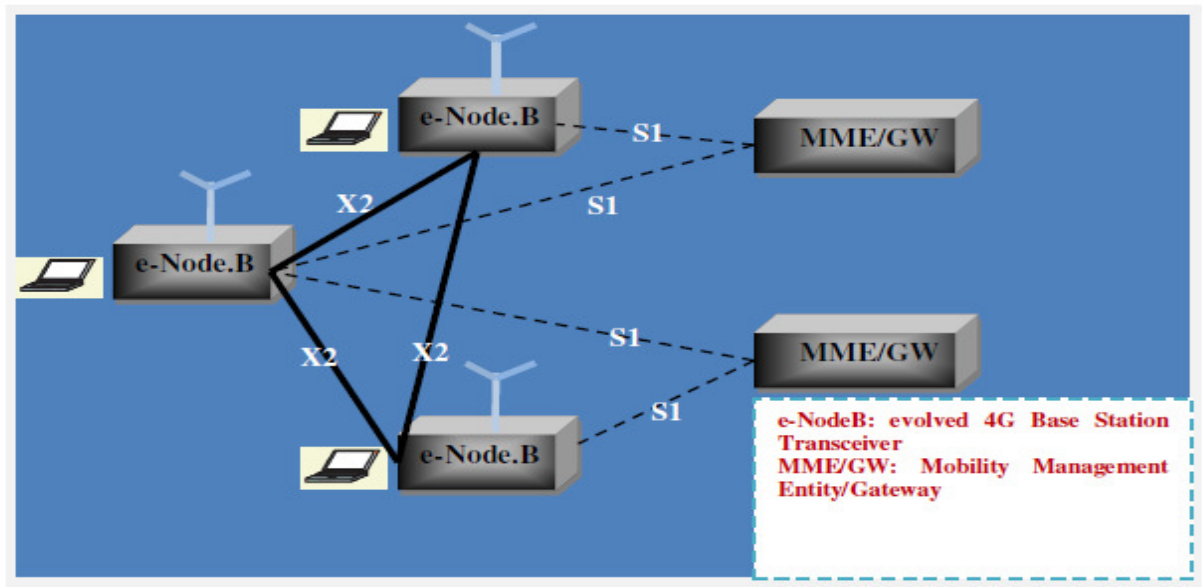

Figure 4: The LTE network architecture.

All the interfaces are based on IP protocols. The eNBs are connected to the MME/GW entity by means of an S1 interface and interconnected together by means of an X2 interface. The LTE architecture includes two logical gateway entities: the serving gateway (S-GW) and the packet data network gateway (P-GW). The S-GW forwards and receives packets to and from the eNB serving the UE. The P-GW interfaces with external packet data networks (PDNs) and performs several IP functions such as address allocation, policy enforcement, packet filtering and routing. The MME is a signaling only entity where user IP packets do not go through. The network capacity for signaling and traffic can grow independently as an advantage of a separate network entity for signaling and traffic.

The Evolved Packet Switched System (EPS) consists of an Evolved Packet Core (EPC) and Evolved UTRAN (E-UTRAN), Figure 5, and provides IP connectivity between a UE and an external packet data network. E-UTRAN consists of eNBs and provides the E-UTRA user plane and control plane protocol terminations towards the UE. The EPC also contains other types of nodes such as Policy and Charging Rules Function (PCRF) responsible for quality-of-service (QoS) handling and charging, and the Home Subscriber Service (HSS) node, a database containing subscriber information. 
International Journal of Next-Generation Networks (IJNGN) Vol.4, No.3,September 2012

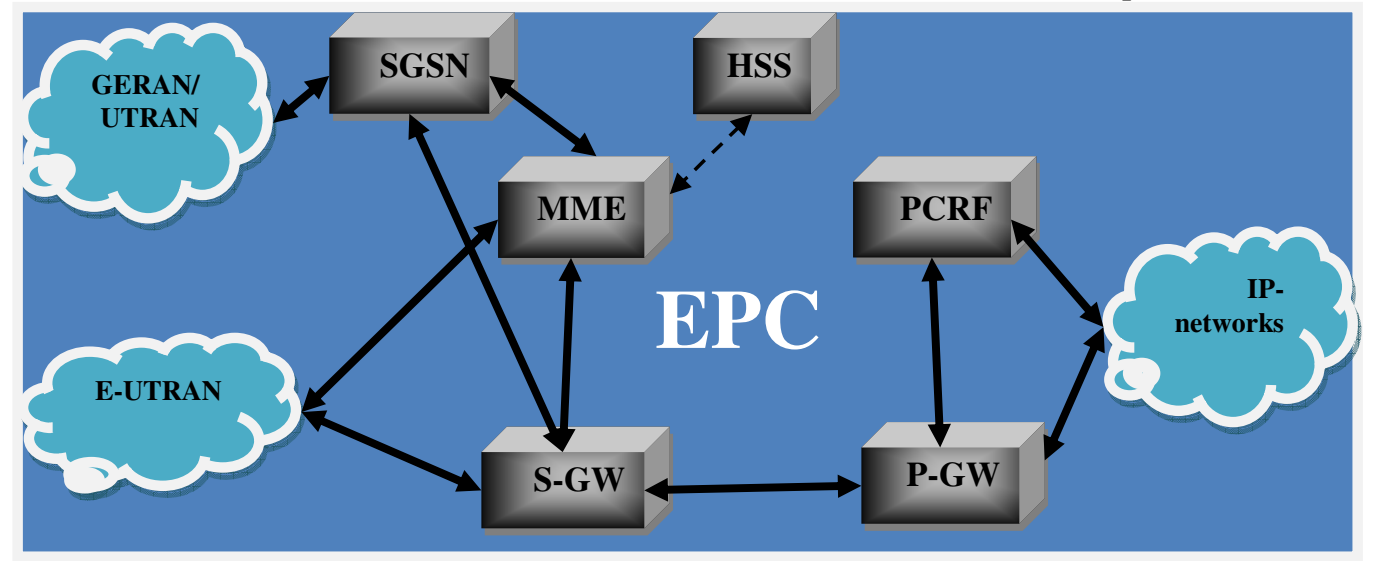

Figure 5: Evolved Packet Core Key Entities.

\subsubsection{Mobile WiMAX (IEEE 802.16e) [25]}

The initial 802.16 specification was designed for line-of-sight operation in the $10-60 \mathrm{GHz}$ frequency range. The next revision, 802.16a, introduced support for non-line-of-sight operation with focus on the $2-11 \mathrm{GHz}$ frequency range, but still targeting the fixed-wireless-access application. Support for mobility was introduced as part of IEEE 802.16e, finalized in December 2005. 802.16e is the basis for all currently commercial mobile-data networks based on the 802.16 technology. Part of 802.16e has also, at a relatively late stage (2007), been approved by the ITU as an additional IMT- 2000 technology in parallel with WCDMA/HSPA, TD-SCDMA, and CDMA2000/1xEV-DO. The 802.16e-based IMT-2000 technology is only supporting TDD operation and is, in the ITU, referred to as IMT-2000 OFDMA TDD WAN.

Mobile WiMAX is based on OFDM transmission supporting different bandwidths for both uplink and downlink. Adaptive modulation and coding ("link adaptation") can be used to adjust the modulation scheme and coding rate, and thus the data rate, to match the instantaneous channel conditions.

\subsection{Fourth Generations Systems}

The 4G IMT-advanced cellular and wireless systems comprise mainly LTE advanced [26] (3GPP family) and Mobile WiMAX advanced (IEEE family, 802.16m) [27]. Both LTE-advanced and IEEE $802.16 \mathrm{~m}$ standards further increases data rates and enhances system spectral efficiency and also supports compatibility with their respective earlier releases. The main features of both are analyzed as follows:

\subsubsection{LTE advanced}

LTE release 10, also referred to as LTE-Advanced, is claimed to be the true $4 \mathrm{G}$ evolution step. Earlier releases of LTE are included as integrated parts of LTE release 10, providing a more straightforward backwards compatibility and support of legacy terminals, for example. The main requirement specification for LTE advanced as approved in [26] are: 
International Journal of Next-Generation Networks (IJNGN) Vol.4, No.3,September 2012

- Peak Downlink data rate: 1 Gbps, Peak Uplink data rate: 500 Mbps.

- Transmission bandwidth: Wider than approximately $70 \mathrm{MHz}$ in DL and $40 \mathrm{MHz}$ in UL.

- User throughput at cell edge 2 times higher than that in LTE.

- Average user throughput is 3 times higher than that in LTE.

- Spectrum efficiency 3 times higher than that in LTE; Peak spectrum efficiency Downlink: 30 bps/Hz, Uplink: 15 bps/Hz.

- Mobility: Same as that in LTE.

- Coverage should be optimized or deployment in local areas/micro cell environments with Inter Site Distance (ISD) up to $1 \mathrm{~km}$.

\subsubsection{Mobile WiMAX advanced, IEEE 802.16 m}

The latest step taken within the IEEE 802.16 community is the development of the $16 \mathrm{~m}$ version of the specification (IEEE $802.16 \mathrm{~m}$ ), a key goal of which was to ensure compliance with the ITU requirements on IMT-Advanced [28]. In October 2010, $802.16 \mathrm{~m}$ was approved by the ITU as a second IMT-Advanced-compliant technology under the name Wireless MAN-Advanced. Thus, similar to LTE, IEEE $802.16 \mathrm{~m}$ can also be seen as a fully compliant 4G technology. However, in contrast to LTE-Advanced being an evolution of LTE release $8 / 9,802.16 \mathrm{~m}$ is not a direct evolution of 802.16e; it does not add new complementary features to extend the performance and capabilities of the $802.16 \mathrm{e}$ radio-access technology. Rather, $802.16 \mathrm{~m}$ is, in many respects, a new radio-access technology although retaining several of the basic characteristics of $802.16 \mathrm{e}$, including the basic OFDM numerology.

This also implies that $802.16 \mathrm{e}$ and $802.16 \mathrm{~m}$ can coexist on the same carrier by means of time multiplexing the two radio-access technologies within the $802.16 \mathrm{e}(5 \mathrm{~ms})$ frame structure. Technology wise, $802.16 \mathrm{~m}$ introduces many features similar to LTE release 10, including the use of multi-carrier transmission (carrier aggregation) for bandwidths beyond $20 \mathrm{MHz}$ and support for relaying functionality. $802.16 \mathrm{~m}$ also introduces substantially shorter subframes of length roughly $0.6 \mathrm{~ms}$ to reduce hybrid- ARQ round-trip time and, in general, allow for reduced latency over the radio interface. Regarding the resource assignment, $802.16 \mathrm{~m}$ does not support the resource mapping schemes specified for $802.16 \mathrm{e}$. Rather, $802.16 \mathrm{~m}$ introduces physical resource units consisting of a number of frequency- contiguous subcarriers during one subframe, very similar to the LTE resource blocks. Actually, the number of subcarriers in a resource unit equals 18 which, together with the subcarrier spacing of $10.94 \mathrm{kHz}$, results in a resource unit with a bandwidth that is very similar to the LTE resource-block bandwidth of $180 \mathrm{kHz}$. Above the physical resource units are logical resource units that can be distributed or localized, where distributed resource units are used for cases when frequency diversity is used. Once again, this is very similar to LTE localized and distributed resource mapping. Taking into account the many similarities between LTE and $802.16 \mathrm{~m}$, it is not surprising that performance evaluations indicate similar performance of the two radio-interface technologies. Thus, similar to LTE, $802.16 \mathrm{~m}$ also fulfils all the requirements for IMT-Advanced as defined by the ITU.

\section{5G Mobile Communications}

The evolution of LTE does not end with LTE advanced (release 10) rather continues to evolve into further releases. Each new release will further enhance system performance and add new 
International Journal of Next-Generation Networks (IJNGN) Vol.4, No.3,September 2012

capabilities with new application areas. Some of the additional applications, benefiting from mobile connectivity, are home automation, smart transportation, security, and e-books, ...etc.

\subsection{Why is there a need for $\mathbf{5 G}$ ?}

The major difference, from a user point of view, between current generations and expected 5G techniques must be something else than increased maximum throughput; other requirements include:

- Lower battery consumption.

- Lower outage probability; better coverage and high data rates available at cell edge.

- Multiple concurrent data transfer paths.

- Around $1 \mathrm{Gbps}$ data rate in mobility.

- More secure; better cognitive radio/SDR Security.

- Higher system level spectral efficiency.

- Worldwide wireless web (WWWW), wireless-based web applications that include full multimedia capability beyond $4 \mathrm{G}$ speeds.

- More applications combined with artificial intelligent (AI) as human life will be surrounded by artificial sensors which could be communicating with mobile phones.

- Not harmful to human health.

- Cheaper traffic fees due to low infrastructure deployment costs.

$5 \mathrm{G}$ is to be a new technology that will provide all the possible applications, by using only one universal device, and interconnecting most of the already existing communication infrastructures. The $5 \mathrm{G}$ terminals will be a reconfigurable multimode and cognitive radio- enabled. It will have software defined radio modulation schemes. All the required reconfigurable software should be downloaded from the Internet on the run. The $5 \mathrm{G}$ mobile networks will focus on the development of the user terminals where the terminals will have access to different wireless technologies at the same time and will combine different flows from different technologies. Besides, the terminal will make the final choice among different wireless/mobile access network providers for a given service. The $5 \mathrm{G}$ core is to be a Re-configurable, Multi-Technology Core. The core could be a convergence of new technologies such as nanotechnology, cloud Computing and cognitive Radio, and based on All IP Platform. These new technologies and the above mentioned requirements pose the following challenges toward $5 \mathrm{G}$ development:

\subsection{G Main Development Challenges:}

\subsubsection{Cognitive Radio (CR)-New ways of Using Spectrum}

New mobile generations are typically assigned new frequency bands and wider spectral bandwidth per frequency channel, but there is little room for new frequency bands or larger channel bandwidths. This is because spectrum has been and will continue to be a scarce resource for the mobile-communication industry. Historically, up until now, the mobile industry has relied on spectrum dedicated for mobile communication and licensed to a certain operator. However, in situations where licensed spectrum is not available, other possibilities for increasing the spectrum availability are of interest. This could include the use of unlicensed spectrum, or secondary 
International Journal of Next-Generation Networks (IJNGN) Vol.4, No.3,September 2012 spectrum primarily used for other communication services, as a complement to operation in the licensed spectrum. Broadcast spectrum not used (in some areas) is often referred to as "white space" [29]. Related to this is the concept of cognitive radio [30]. However, the applicability of cognitive radio to cellular communication is a relatively new area and further studies are required to assess the feasibility and impact of such usage. Cognitive radio technology allows different radio technologies to share the same spectrum efficiently by adaptively finding unused spectrum and adapting the transmission scheme to the requirements of the technologies currently sharing the spectrum. This dynamic radio resource management is achieved in a distributed fashion, and relies on software-defined radio.

\subsubsection{Software Defined Radio (SDR)-Reconfigurability enabler}

Software Defined Radio (SDR) [31] benefits from today's high processing power to develop multiband, multi-standard base stations and terminals. Although in future the terminals will adapt the air interface to the available radio access technology, at present this is done by the infrastructure. Several infrastructure gains are expected from SDR. For example, to increase network capacity at a specific time (e.g. during festivals or sport events), an operator will reconfigure its network adding several modems at a given Base Transceiver Station (BTS). SDR makes this reconfiguration easy. In the context of the expected 5G systems, SDR will become an enabler for terminal and network reconfigurability through software download. For manufacturer, this can be a powerful aid to providing multi-standard, multi-band equipment with reduced development effort and costs.

\subsubsection{Reconfigurable-Interoperability between several types of wireless access network}

Seamless interoperability among heterogeneous networks represents the corner stone for the success of $5 \mathrm{G}$ systems with different evolving access technologies. A novel solution that ensures interoperability between several types of wireless access network is given by the developing IEEE 802.21 standard [32]. The IEEE 802.21 is focused on handover facilitation between different wireless networks in heterogeneous environments regardless of the type of medium. The standard names this type of vertical handover as Media Independent Handover (MIH). The goal of IEEE 802.21 is to ease the mobile nodes' usage by providing uninterrupted handover in heterogeneous networks. The heart of the 802.21 framework is the Media Independent Handover Function (MIHF), responsible for communication with different terminals, networks and remote MIHFs, which will have to be implemented in every IEEE 802.21 compatible device (in either hardware or software). The interest that exist both in academia and industry shows that IEEE 802.21 may be the key enabler for seamless vertical handover and transparent roaming in heterogeneous networks. IEEE 802.21 standard is expected to make a major contribution towards the reconfigurable interoperability aspect of $5 \mathrm{G}$ wireless and cellular communications systems. The reconfigurable interoperability offers network providers with a possibility to choose, with minimal investments, between alternative wireless access networks. The selection could be made based on several criteria such as:

- Comparison between the availability of access resources and specific service requirements (e.g. channel state, outage probability, vertical handover probability, users' QoS requirements, context awareness etc.). 
International Journal of Next-Generation Networks (IJNGN) Vol.4, No.3,September 2012

- Load balancing and sharing between different spatially coexisting wireless networks.

- Efficient spectrum sharing.

- Congestion control.

In general, the main requirements for interoperability that need to be taken into consideration are as follows:

- Initial Network Selection (INS); INS is one of the basic functions of interoperability process between heterogeneous networks. A clever selection of a suitable network by users would result in lower blocking probability, higher capacity and enhanced QoS. These benefits can be achieved only if INS enables an efficient use of network resources. As a result, users can select the most appropriate network with an enhanced QoS with respect to the desired service requirements. The achievement of these enhancements depends on the integration architecture of the two technologies and on developing efficient INS mechanisms and criteria.

- Mobility support (vertical or internetwork Handover); once a network has been selected, the user is subject to change the initially selected network according to various conditions; hence the importance of an efficient Inter-Network Handover (INH) criteria and mechanisms arises. Decision for INH could be based on an evaluation of a cost function that covers all possible inter-network handover's key factors. Decision for both INS and INH as cost function based radio access selection and inter-radio access handover is clearly discussed in [33].

- Partnership or roaming agreements between different interoperating networks' operators; operators should give the user the same benefits as if the interoperating was handled within one network operator.

- Handling subscriber billing and accounting between roaming systems.

- Identification of subscriber should be done as if it is in a pure one system environment.

\subsubsection{Adaptive Coupling-Reconfigurable Integration}

Depending on the level of integration that is required between available radio access technologies, a variety of approaches can be taken for effective interoperability. On the one hand, if the integration between different technologies is tight, the provisioning of the service is more efficient and network selection as well as the vertical handover process is faster. However, a high level of integration requires considerable effort in the definition of interfaces and mechanisms able to support the necessary exchange of data and signaling between different radio access networks. Moreover, tight coupling suffers from potential of load congestion when one network full load is immersed on the other. On the other hand, if the integration between different technologies is loose, the delay of handover process is significant. On the positive side, loose coupling allows for the flexibility and independence of implementing individually different mechanisms within each network. Besides, it eases the gradual deployment of one network with no or little modification on the other network(s). Adaptive coupling is a new proposed mechanism that adaptively changes coupling level from open, loose to tight and even very tight according to networks' load status and delay constraints. 
International Journal of Next-Generation Networks (IJNGN) Vol.4, No.3,September 2012

\subsubsection{Network Energy Efficiency}

Low energy consumption for mobile terminals has been an important requirement since the emergence of hand-held terminals roughly 25 years ago. The driving force has been the reduction in battery size and improved battery time. Today, reduced energy consumption also in the radioaccess network is receiving increased attention; the cost of energy is a far from negligible part of the overall operational cost for the operator. With sufficiently low energy consumption, reasonably sized solar panels could be used as power source, instead of the diesel generators commonly used today. Nevertheless, the future evolution of cellular systems should further strive for minimizing transmission of signals strictly not needed.

\subsubsection{Machine-Type Communication}

With the increased availability of mobile broadband, connectivity has become a realistic option for machine-type communication. Machine-type communication spans a wide range of applications, from massive deployment of low-cost battery-powered sensors to remote-controlled utility meters, to surveillance cameras. Many of these applications can be handled by 4G systems already; communicating with a surveillance camera, for example, is not significantly different from uploading a file, in which case high data rates are paramount. However, other applications may not require transmission of large amounts of data or low latency, but rather pose challenges in terms of a vast amount of devices connecting to the network. Handling such a large number of devices is likely to be a challenge mainly for the core network, but improvements in the area of connection setup and power efficient handling of control signaling in the radio-access network may be of great interest for future $5 \mathrm{G}$ mobile communications.

\subsubsection{Nanotechnology}

Nanotechnology is the application of nanoscience to control process on nanometer scale; between 0.1 and 100nm. The field is also known as molecular nanotechnology (MNT) where MNT deals with control of the structure of matter based on atom-by-atom and molecule by molecule engineering. Nanotechnology is considered as the next industrial revolution, and the telecommunications industry will be radically transformed by it in a few years. As the future applications will require more memory and computing power to offer higher data rates, current technologies can not resolve these challenges. Fortunately, nanotechnology could provide effective solutions for power efficient computing, sensing, memory enlargement, and humanmachine interaction, [34], [35]. Nanotechnology will have considerable impacts on both mobile device as well as core network as follows:

- The mobile device has become more than a communication device in modern world; computation and communication are ready to serve the user in an intelligent way. Mobile devices together with the intelligence, embedded in human environments, will create a new platform that enables ubiquitous sensing, computing, and communication. With nanotechnology mobile phones can act as intelligent sensors that have applications in many industries, among them transportation, communications, medicine and safety.

- The core network requires high speed and a reliable capacity to manipulate and interoperate increasing number of heterogeneous access technologies. At present, nanotechnologies are used in Digital Signal Processing (DSP) Fabrication, introducing new perceptions in DSP designing that increases the overall system speed \& capacity. 
International Journal of Next-Generation Networks (IJNGN) Vol.4, No.3,September 2012

\subsubsection{All IP Network}

The All-IP Network (AIPN) is an evolution of the 3GPP system to fulfill the increasing demands of the cellular communications market. It is a common platform valid for all sorts of radio access technologies. AIPN focused primarily on the enhancements of packet switched technology but now it provides a continued evolution and optimization in terms of both performance and cost. The key benefits of AIPN architecture includes a variety of different access systems' provision, lower costs, universal seamless access, increased user-satisfaction and reduced system latency. But with the advantages of IP come some dangers: as data flow more freely and the internet is open not only to developers but also to all manner of criminals and viruses, developers and operators face new security challenges which should be solved properly.

\subsubsection{Cloud computing}

"Cloud computing is a model for enabling ubiquitous, convenient, on-demand network access to a shared pool of configurable computing resources (e.g., networks, servers, storage, applications, and services) that can be rapidly provisioned and released with minimal management effort or service provider interaction..." a definition from [36]. Hence, cloud computing is a technology that uses the internet and central remote server to maintain data and applications. In 5G networks this central remote server could be a content provider. Cloud computing allows consumers and business to use applications without installation and access their personal files at any computer with internet access. The same concept is going to be used in multi-core technology where the user tries to access his private account form a global content provider through cloud computing.

\subsection{A proposed 5G Network Architecture}

Terminals and network components are dynamically reconfigured (adapted) to new situation. Network operators use the reconfigurability to introduce value-added services more easily. Reconfigurability is based on cognitive radio. Cognitive radio technologies includes the ability of devices to determine their location, sense spectrum used by neighboring devices, change frequency, adjust output power, and even alter transmission parameters and characteristics. A cognitive radio is a transceiver that is able to understand and react to its operating environment. Thus cognitive radio concerns mobile devices and networks which are computationally intelligent about radio resources and related communications to detect user communication needs and provide wireless services appropriate to those needs. Hence, the radio is aware and cognitive about changes in its environment and responds to these changes by adapting operating characteristics in some way to improve its performance.

\subsubsection{Reconfigurable-Multimode-Terminal}

The $5 \mathrm{G}$ potential will require the design of a single wireless user terminal able to autonomously operate in different heterogeneous access networks. A fully reconfigurable terminal changes its communication functions depending on network and/or user demands. Moreover, this terminal will have to exploit various surrounding information such as communication with navigation and localization systems and communications with weather forecast and emergency systems in order to provide richer user services. However, the richness of the services will necessitate higher bit rates, which will be the main driving factor towards broadband multimedia development. Also, 
International Journal of Next-Generation Networks (IJNGN) Vol.4, No.3,September 2012 the terminal awareness will put strong emphasis on the concept of cognitive radio and cognitive algorithms for stand-alone terminal reconfigurability and interoperability. The design of a reconfigurable multimode cognitive user terminal faces several problems; there must be reductions in cost, size, power consumption and circuit complexity that will lead to a reconfigurable user terminal which is cheap, wearable and conformant. This could be achieved easily with the huge capability of nanotechnology. In addition, sophisticated autonomous network tracking and dynamic network selection algorithms must be developed. Users' terminals reconfigure themselves according to the selected network features.

A software reconfigurable transceiver implements its communication functions as programs running on a suitable processor where different transmitter/receiver algorithms, which usually describe transmission standards, are implemented in software. The terminal reconfiguration enables it to be connected to different radio access technologies; ranging from 2G/GERAN to 3G/UTRAN and 4G/EUTRAN in addition to 802.11x WLAN and 802.16x WMAN. Other standards are also enabled such as IS/95, EV-DO, CDMA2000...etc. Interoperability processcriteria and mechanisms- as illustrated in subsection 3.2.3 enables both terminal and reconfigurable multi-technology core to select from the above heterogeneous access systems. Figure 6 shows a reconfigurable transceiver in both terminal and base stations. It can be reconfigured via a control bus supplying the processing units with the parameters downloaded from remote re-configuration database via a predefined broadcasting download channel. Such a configuration guarantees that the transmission can be changed instantaneously if necessary (e.g., for inter-netwok handover). Communication standard are parameterized as a set of documents that comprehensively describe all functions of a radio system. A reconfigurable transceiver is built in open-architecture and based on radio system software [37]. Functional modules of the radio system such as modulation/demodulation, signal generation, coding and link-layer protocols are implemented on generic reprogrammable hardware platforms using programmable modules such as Digital Signal Processors (DSPs), Field Programmable Gate Arrays (FPGAs) and MicroControllers (MCs), and analog RF modules.

The RF front-end functions as the transmitter and receiver for the RF signal received via the antenna. On the receive path, it down-converts the RF signal to IF signal for further processing in the IF section. On the transmit path, it performs up-conversion to convert the IF signal to RF signal follow by power amplification. The IF section is responsible for analog-to-digital conversion (ADC) and digital-to-analog conversion (DAC) on the receive path and the transmit path, respectively. The digital down converter (DDC) and digital up-converter (DUC) that proceeds and precedes the ADC and DAC respectively, jointly assume the functions of a modem. The baseband section performs baseband operations such as connection setup, equalization, frequency hopping, timing recovery and correlation. In a reconfigurable transceiver, the baseband processing is designed to be software programmable. On top of that, the DDC and DUC modules in the IF section are also programmable. The link layer protocols, modulation and demodulation operations are implemented in software. Thus, the operational mode of the transceiver can be changed or augmented post-manufacturing using software. An ideal reconfigurable transceiver is one that is programmable up till the RF section, i.e., capable of performing high speed and power efficient analog-to-digital conversion and vice-versa right at the antenna. However, supporting the required digital bandwidth, dynamic range and sampling rate for efficient implementation of programmable RF section is still a challenge for more development. 


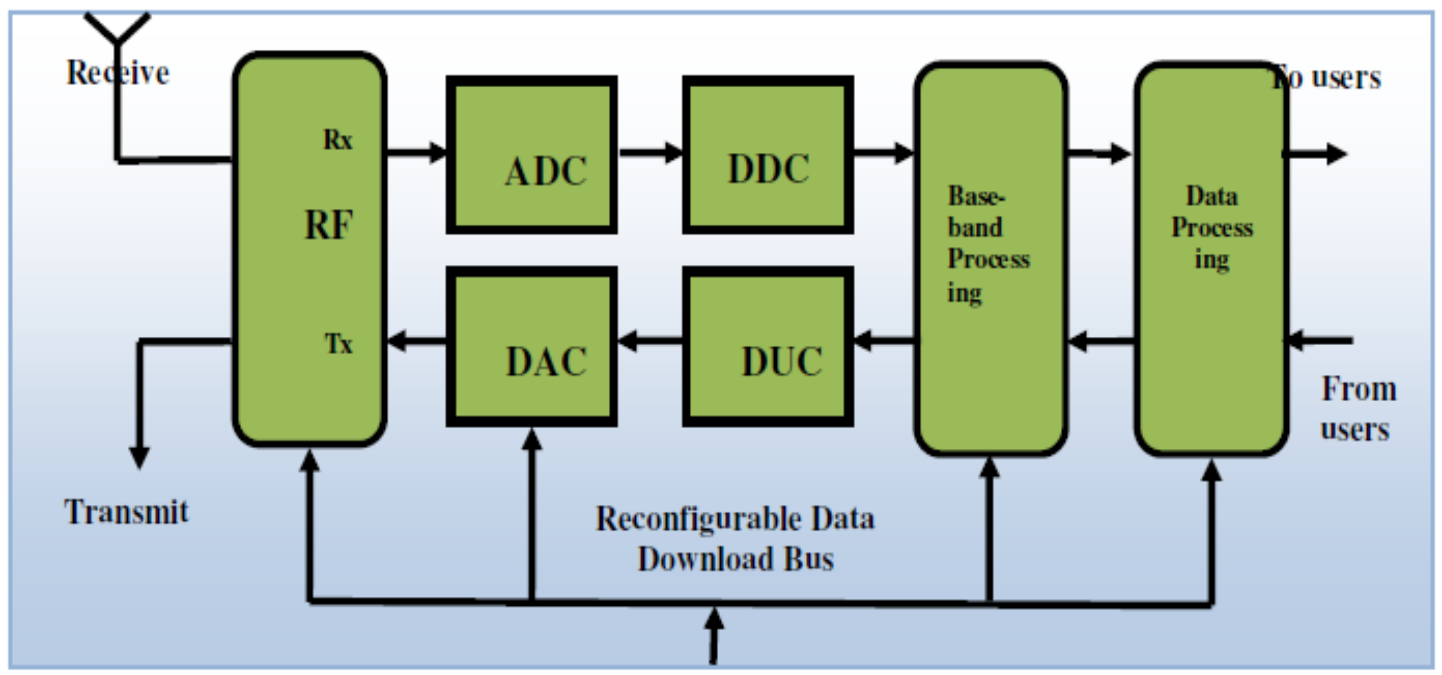

Figure 6: High Level Structure of Reconfigurable Transceiver

\subsubsection{Reconfigurable Multi-Technology Core}

The main challenge for a reconfigurable multi-technology core is to deal with increasing number of different radio access technologies based on solid interoperability criteria and mechanisms. The core is a convergence of the aforementioned nanotechnology, cloud computing and cognitive radio, and based on All IP Platform. Core reconfigurability could be a self-adaptation to a dynamically-changing environment or mission oriented adaptation to meet a given set of mission requirements with the aim of improving service delivery and spectrum utilization. Core changes its communication functions depending on network status and/or user demands. Reconfigurability could be in both software and hardware. Hardware reconfiguration is mainly performed by operators; adding additional equipments to increase network capacity at a specific time. However, in software reconfiguration and with the power of SDR, network is dynamically reconfigurable, which means that the programs (running on the reconfigurable processing elements) as well as the communication links between the processing elements are configured at run-time. Different processing elements are used for different purposes. The general purpose processors are fully programmable to perform different computational tasks. Figure 7 shows a high level structure of reconfigurable core network as an evolution of the Evolved Packet Core (EPC) of 4G network. Local Reconfiguration Database (LRD) attached to Reconfiguration Data models (RDM) are connected to gateway entities via Reconfiguration Control and Management unit (RCM). RCM is also connected to Cloud Computing Resources (CCR) to link core network with Remote Reconfiguration Database (RRD). Basic entities of the EPC are enhanced with reconfigurable capability such as Reconfigurable Serving Gateway (RS-GW) and Reconfigurable Packet data network Gateway (RP-GW). RS-GW is linked to different access technologies via Reconfigurable-Interoperability Control (RIC) unit. RIC controls inter-operability process amongst heterogeneous access technologies and enables RS-GW to forward and receive packets to and from the selected base station/eNB serving the UE. To serve the user with All IP based mobile applications and services, RP-GW interfaces, via CCR, with the Internet and other packet 
International Journal of Next-Generation Networks (IJNGN) Vol.4, No.3,September 2012 data networks (PDNs). The Mobility Management Entity (MME), as a signaling only entity, links RS-GW to Home Subscriber Database (HSD) node.

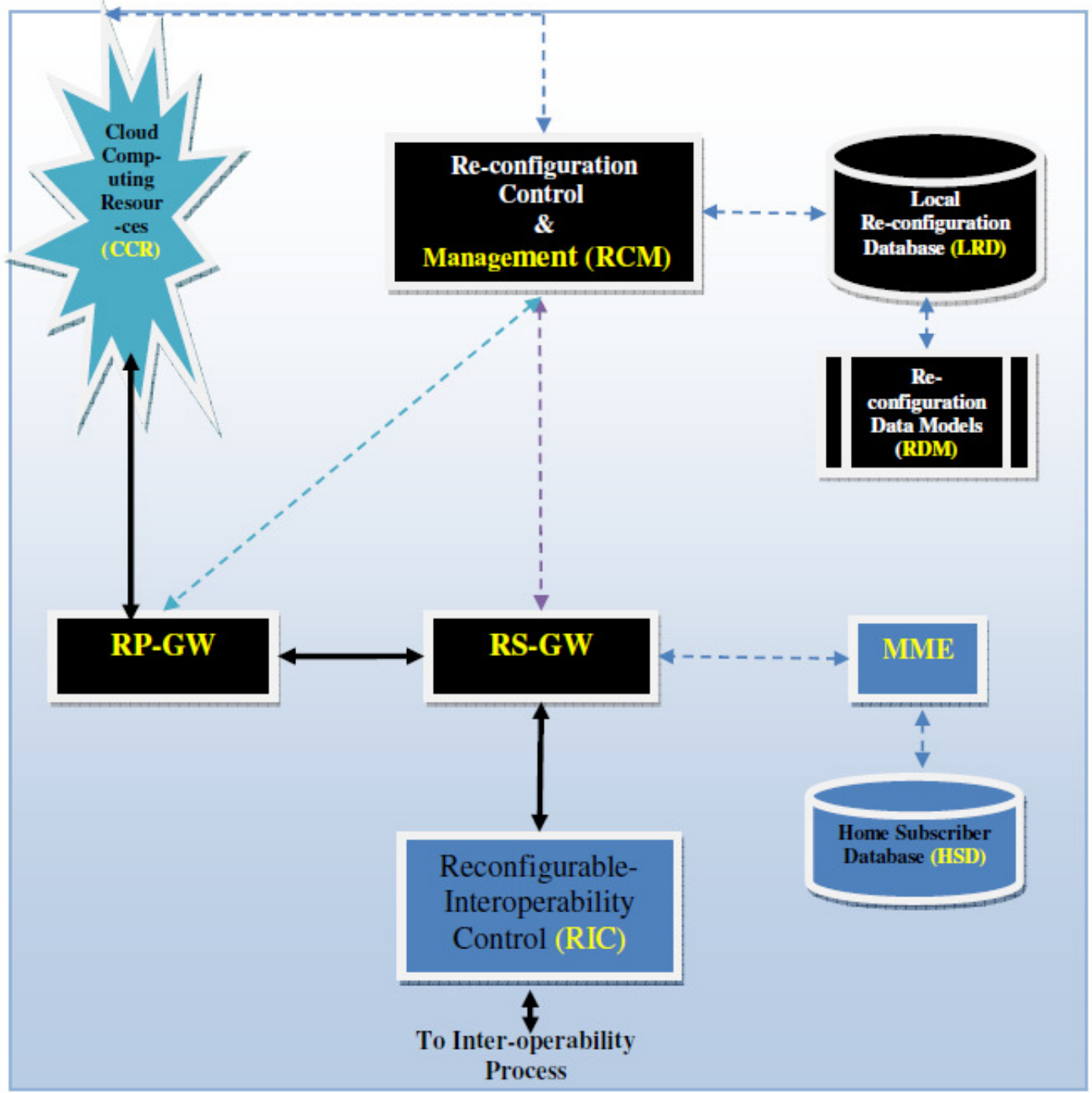

Figure 7: High Level Structure of Reconfigurable Multi-Technology Core

Figure 8 shows a proposed 5G Network Architecture. All IP based mobile applications and services such as Mobile portals, Mobile commerce, Mobile health care, Mobile government, Mobile banking and others, are offered via Cloud Computing Resources (CCR). CCR links the Reconfigurable Multi-Technology Core (RMTC) with remote reconfiguration data from RRD attached to Reconfiguration Data models (RDM). RMTC is connected to different radio access technologies; ranging from 2G/GERAN to 3G/UTRAN and 4G/EUTRAN in addition to $802.11 \mathrm{x}$ WLAN and 802.16x WMAN. Other standards are also enabled such as IS/95, EV-DO, CDMA2000...etc. Interoperability process-criteria and mechanisms enable both terminal and RMTC to select from the above heterogeneous access systems. 


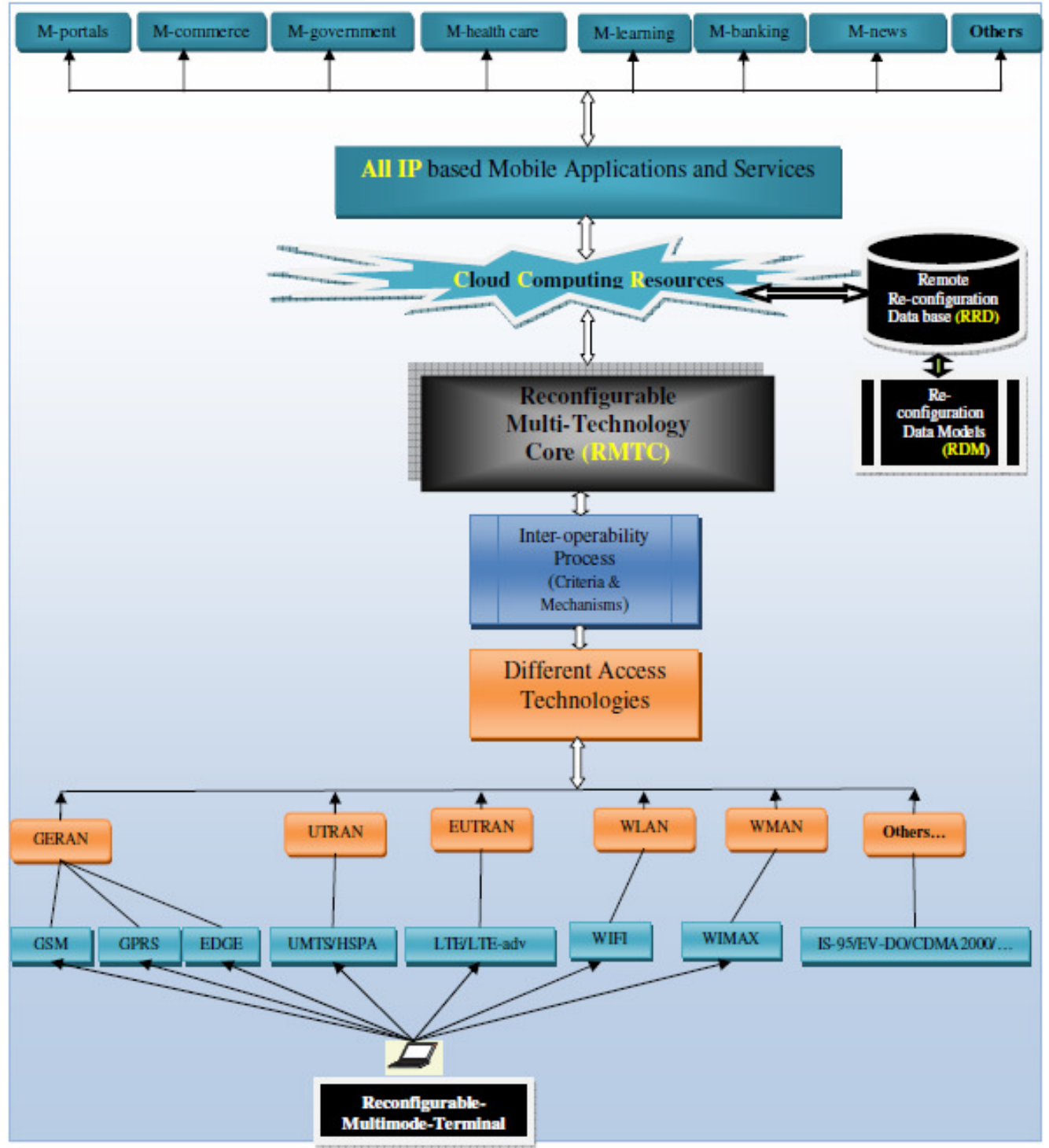

Figure 8: A proposed 5G Network Architecture

\section{Conclusion}

The paper was focused on building $5 \mathrm{G}$ reconfigurable mobile system benefiting from latest technologies such as cognitive radio, SDR, nanotechnology, cloud computing and based on All IP Platform. The goal was for both terminal and core network to dynamically reconfigured (adapted) to new situation and changes their communication functions depending on network and/or user demands. The paper discussed 5G main development challenges and clarified the necessity for 5G. It also reviewed in brief the evolution of wireless and cellular systems focusing on four main key factors: radio access, data rates, bandwidth and switching schemes in addition to change in 
International Journal of Next-Generation Networks (IJNGN) Vol.4, No.3,September 2012

network architecture. The 3G transitional cellular and wireless systems toward $4 \mathrm{G}$ and the true 4G IMT-advanced systems were thoroughly presented

\section{REFERENCES}

[1] URL: www.gsmworld.com/technology/gprs

[2] The Evolution of EDGE (PDF file - Ericsson White Paper) 285 23-3107 Uen Rev. (C Ericsson AB 2007

[3] URL: http://www.umts-forum.org

[4] 3GPP Specifications, TR 21.101 URL: http://www.3gpp.org/article/release-5

[5] UTRA-UTRAN Long Term Evolution (LTE) URL: http://www.3gpp.org/article/lte

[6] Erik Dahlman et al., "4G LTE/LTE-Advanced for Mobile Broadband”, Elsevier, 2011

[7] URL: http://www.wi-fi.org

[8] URL: http://www.wimaxforum.org

[9] SAYAN KUMAR RAY, Fourth Generation (4g) Networks: Roadmap- Migration to the Future IETE Technical Review, Vol 23, No 4, July-August 2006, pp 253-265

[10] URL: http://www.3gpp.org

[11] URL: http://www.wimaxforum.org

[12] URL:http://www.itu.int/ITU-R/index.asp?category=information\&rlink=rhome\&lang=en

[13] GSM Forum Europe, http://www.gsm-forum.eu/

[14] The Evolution of EDGE (PDF file - Ericsson White Paper) 285 23-3107 Uen Rev. (C Ericsson AB 2007

[15] http://www.itu.int/newsroom/press_releases/2007/30.html

[16] http://www.umtsworld.com/technology/overview.htm

[17] Kreher and Ruedebusch, UMTS Signaling: UMTS Interfaces, Protocols, Message Flows and Procedures Analyzed and Explained (Wiley 2007), ISBN 978-0470065334

[18] Theodore S. Rappaport, (Wireless Communications-Principles and Practices), Second Edition 2004

[19] http://en.wikipedia.org/wiki/High-Speed_Uplink_Packet_Access

[20] URL: http://hsopa.org/

[21] UTRA-UTRAN Long Term Evolution (LTE) http://www.3gpp.org/article/lte

[22] URL: http://www.wimaxforum.org

[23] 3GPP TSG RAN TR 25.913 v7.3.0, Requirements for Evolved Universal Terrestrial Radio Access (UTRA) and Universal Terrestrial Radio Access Network (UTRAN).

[24] FAROOQ KHAN, LTE for 4G Mobile Broadband, Cambridge University Press, 2009

[25] IEEE Std 802.16e-2005,Air Interface for Fixed and Mobile BroadbandWirelessAccess Systems.

[26] 3GPP TSG RAN TR 36.913 v8.0.0, Requirements for Further Advancements for E-UTRA (LTEAdvanced).

[27] IEEE 802.16m-07/002r4, TGm System Requirements Document (SRD).

[28] ITU-R, ITU paves way for next-generation $4 \mathrm{G}$ mobile technologies; ITU-R IMT-advanced $4 \mathrm{G}$ standards to user new era of mobile broadband communications, ITU Press Release, 21 October 2010.

[29] M. Nekovee, A survey of cognitive radio access to TV white spaces, Int. J. Digi. Multimed. Broadcast. (2010) 1-11.

[30] S. Haykin, Cognitive radio: Brain-empowered wireless communications, IEEE J. Sel. Area. Comm. 23 (2) (February 2005) 201-220.

[31] URL: http://www.sdrforum.org

[32] IEEE 802.21: Media Independent Handover, URL: http://www.ieee802.org/21/

[33] Zhuo Sun and Wenbo Wang "Investigation of Cooperation Technologies in Heterogeneous Wireless Networks", Journal of Computer Systems, Networks, and Communications, Volume (2010), Article ID 413987.

[34] Ermolov V. et al. "Significance of Nanotechnology for future wireless devices and Communications", The 18th Annual IEEE International Symposium on PIMRC'07. 
International Journal of Next-Generation Networks (IJNGN) Vol.4, No.3,September 2012

[35] R.K.Jain, Risal Singh, "Role of Nanotechnology in future wireless and communication systems", National seminar proceeding, Academy of Business \& Engineering Science Ghaziabad, pp-19-28, 1617 th January 2009.

[36] Peter Mell and Timothy Grance, "The NIST Definition of Cloud Computing” US National Institute of Standards and Technology Special Publication 800-145, September 2011.

[37] Friedrich K. Jondral, "Software-Defined Radio-Basics and Evolution to Cognitive Radio" EURASIP Journal on Wireless Communications and Networking 2005:3, 275-283

\section{Biography}

Currently working as associate professor at the University of Palestine, Dr. Mousa was granted the PhD on "4G Cellular and WLAN Inter-working Networks" from the National Technical University of Athens, Dept. of Electrical \& Computer Engineering in 2004. Dr. Mousa obtained a D.E.A (equivalent Master degree) in Digital Telecommunication Systems from \{Ecole Nationale Supérieure des Télécommunications/PARIS\} in 1996 and the (B.Sc.) of Electronic Engineering from \{Middle East Technical University/Ankara\} in 1992. 